\title{
Investigating neutropenia and its outcomes in newborns admitted to the Neonatal Intensive Care Unit Center in the Southwest of Iran (Imam Khomeini Hospital, Ahvaz).
}

\author{
Arash Malakian*, Mohammad Raza Aramesh, Masoud Dehdashtiyan, Marzieh Rahnama, Seyyed \\ Mohammad Hassan Aletayeb
}

Department of Paediatrics, Imam Khomeini Hospital, Ahvaz Jundishapur University of Medical Sciences, Ahvaz, Iran

\begin{abstract}
Background: Neutropenia is one of the important risk factors of infection. Neutropenia is associated with various items include mother's blood pressure and hemolytic anemia. The aim of this study was evaluation of neutropenia and its complications in Neonatal Intensive Care Unit (NICU).

Materials and methods: In this analytical cross sectional study, the records of 315 neonates hospitalized in the NICU of Imam Khomeini hospital were studied for the diagnosis of neutropenia. Statistical methods of independent $t$-test and Chi-square were used for the measurement of the date. Analyses were performed by using SPSS version 17.

Results: Nineteen of 315 cases $(6 \%)$ had neutropenia. There was no significant correlation between two genders (73.7\% male and $26.3 \%$ female) in neutropenic neonates. Seven patients $(36.8 \%)$ were dead and $12(63.2 \%)$ were alive $(P$-value $=\mathbf{0 . 0 2})$. Five cases $(26.3 \%)$ were infected and 14 cases $(\mathbf{7 3 . 7 \%})$ had no infection $(P$-value $=0.86)$. In 10 neutropenic cases $(52.6 \%)$ high blood pressure of the mother was observed during pregnancy $(\mathbf{P}$-value $=\mathbf{0 . 0 0})$. Significant difference was observed between birth weight infants and neutropenia $(P$-value $=0.01)$.

Conclusion: Our study showed that neutropenia has a reverse relationship with gestational age and low birth weight. Neutropenia has a direct significant correlation with infant mortality and high maternal hypertension. There was no significant correlation between neutropenia with neonatal infections, Apgar score 5 and the gender of neonate.
\end{abstract}

Keywords: Neutropenia, Infant, Newborn, Sepsis.

Accepted on January 24, 2019

\section{Introduction}

Newborns face many challenges, especially if they are born prematurely. One of these challenges is the difference between the newborn's immune system and the infant's immune system $[1,2]$. Neutropenia is a known disorder in newborns who receive treatment in neonatal intensive care units (NICUs) [3]. In several previous studies, the prevalence of neutropenia in newborns admitted to neonatal intensive care units was reported to be approximately $6 \%$ to $8 \%[2,4]$. The absolute neutrophil count (ANC) can be obtained from a complete blood count (CBC) [3].

By definition, a reduction of ANC to less than 1,500 cubic millimeters is called neutropenia. Mild neutropenia emergences when the number of neutrophils ranges from 1000 to 1500 cubic millimeters, and moderate neutropenia is present when the number of neutrophils ranges from 500 to 1000 cubic millimeters. Finally, severe neutropenia occurs if the number of neutrophils drops to less than 500 cubic millimeters [2,5].
In neutropenic newborns, there is an uncertain relationship between low ANC and the risk of infection. Although the risk of hospital-acquired infection is unlikely in case of $\mathrm{ANCs}>1000 / \mu \mathrm{L}$, it appears that in case of $\mathrm{ANC}<500 / \mu \mathrm{L}$, the risk of hospital-acquired infection increases, especially if neutropenia lasts for more than a few days [3].

Various factors (e.g., maternal blood pressure) can cause neutropenia in newborns. Previous studies have demonstrated that maternal blood pressure can cause neutropenia in newborns and may subsequently lead to impaired function in the newborns' immune systems. Other factors involved in this phenomenon include multiple births, hemolytic diseases, periodic neutropenia, congenital neutropenia (Kostmann syndrome), isoimmune neutropenia and autoimmune neutropenia [6].

Based on the abovementioned points, the researchers decided to conduct a cross-sectional study on newborns admitted to the NICUs to investigate neutropenia and the association of this disorder with the duration of hospitalization and early infection. 


\section{Materials and Methods}

In this cross-sectional descriptive study, the Hospital's archives were searched for existing files, based on a data collection form, to extract information for 315 newborns who were admitted to the NICUs (Level 3) in the Imam Khomeini Hospital in Ahvaz from October 2010 to October 2011. Simple sample selection was used to collected data. White blood cell numbers were counted using the Sysmex tool.

In this study, early neonatal sepsis was defined as having clinical symptoms and positive blood cultures in the first seven days of life. Clinical sepsis was determined based on the presence of 3 or more of the following symptoms: apnea, respiratory problem, cyanosis, tachycardia or bradycardia, impaired tissue perfusion or shock, irritability, lethargy, hypotonia, seizure, abdominal distension, vomiting, nutritional intolerance, food residue in the stomach, hepatomegaly, jaundice without any certain cause, temperature imbalance, petechia or purpura and poor condition of the newborn [5].

Gestational age was determined on the basis of the first day of the last menstruation until the birth of the newborn or the results of sonography in the first month. For the mothers' medical records, it was indicated that the risk of maternal blood pressure during pregnancy was determined in the range of a systolic pressure equal to or greater than $140 \mathrm{~mm} \mathrm{Hg}$ or a diastolic pressure greater than $90 \mathrm{~mm} \mathrm{Hg}$. This blood pressure emerged after the twentieth week of pregnancy and presented with proteinuria equal to or greater than $300 \mathrm{mg}$ at 24 hours [5]. Asphyxia was considered to occur for a five-minute Apgar score less than 5 [2]. Inclusion criteria included the admission of newborns to the NICUs, and exclusion criteria included incomplete information about newborns admitted to the NICUs.

An independent t-test and Chi-square test were used to statistically analyse the data. All statistical analyses were performed at the significance level of $\alpha=0.05$ using SPSS (Version 17).

\section{Results}

In this study, 315 newborns were studied, among whom 296 newborns had a normal rate of neutrophils; 19 newborns were afflicted with neutropenia. Among the newborns with normal neutrophil levels, 190 (64.1\%) were male, and 106 (35.9\%) were female (Table 1). Among the newborns with neutropenia, $14(73.7 \%)$ were male, and 5 were female $(26.3 \%)$. There was no significant relationship between the newborns' gender and the presence of neutropenia ( $p$-value $=0.4$ ).

Table 1. Specifications of newborns under investigation.

\begin{tabular}{llll}
\hline Variable & $\begin{array}{l}\text { Neutropenic } \\
\text { newborns }\end{array}$ & $\begin{array}{l}\text { Non-neutropenic } \\
\text { newborns }\end{array}$ & P-value \\
\hline Gender & & & 0.4 \\
\hline $\mathrm{N}($ Male $)$ & 14 & 190 & \\
\hline $\mathrm{N}($ Female $)$ & 5 & 106
\end{tabular}

\begin{tabular}{|c|c|c|c|}
\hline Birth weight (g) & 1488 & 1929 & 0.01 \\
\hline \multicolumn{4}{|l|}{ Gestational age (week) } \\
\hline$>32$ & 14 & 154 & \\
\hline $32-36$ & 5 & 102 & \\
\hline$<36$ & 0 & 40 & \\
\hline Maternal hypertension & & & 0 \\
\hline $\mathrm{N}$ (Normal) & 9 & 251 & \\
\hline N (High) & 10 & 45 & \\
\hline Five-minute Apgar score & & & 0.1 \\
\hline$>5$ & 2 & 18 & \\
\hline$\leq 5$ & 16 & 278 & \\
\hline $\begin{array}{l}\text { Duration of hospitalization } \\
\text { (day) }\end{array}$ & 12.14 & 9.55 & 0.1 \\
\hline Mortality rate & & & 0.02 \\
\hline Number of deaths & 7 & 48 & \\
\hline Number of recoveries & 12 & 248 & \\
\hline Early sepsis & 5 & 14 & 0.86 \\
\hline \multicolumn{4}{|l|}{ Number of infections } \\
\hline Number of non-infections & 14 & 259 & \\
\hline
\end{tabular}

The mean gestational age of the neutropenic group and newborns with normal neutrophils was 30 and 32 weeks, respectively. The mean birth weight of neutropenic and nonneutropenic newborns was 1488 and 1929 g, respectively. Three neutropenic newborns weighed less than $1000 \mathrm{~g}, 7 \mathrm{of}$ them weighed between 1001 and $1500 \mathrm{~g}$ and 9 weighed between 1501 and $2000 \mathrm{~g}$. Among the non-neutropenic newborns, 25 newborns weighed less than 1000 g, 75 weighed between 1001 and $1500 \mathrm{~g}, 140$ weighed between 1501 and $2500 \mathrm{~g}$ and 55 weighed more than $2500 \mathrm{~g}$. There was a significant relationship between neutropenia and newborns' birth weight $(\mathrm{P}$-value $=0.01)$. Ten neutropenic newborns $(52.6 \%)$ were born from mothers who had gestational hypertension, and 9 newborns (47.4\%) had mothers with normal blood pressure. Among the non-neutropenic newborns, 45 mothers $(15.2 \%)$ had gestational hypertension, and 251 mothers $(84.8 \%)$ had normal blood pressure. There was a significant relationship between neutropenia and gestational hypertension $(\mathrm{P}$-value=0.00). The five-minute Apgar score was less than 2 in two neutropenic newborns, but it was equal to or greater than 5 in 16 newborns. There was no significant correlation between the five-minute Apgar score and neutropenia $(\mathrm{P}-\mathrm{value}=0.1)$. In non-neutropenic newborns, it was found that the five-minute Apgar score was less than 5 in 18 newborns, but it was equal to or greater than 5 in 278 newborns. Five neutropenic newborns had an early neonatal infection, and 37 non-neutropenic newborns had an early neonatal infection. Thus, there was no significant relationship between the groups in this regard $(\mathrm{P}$-value=0.86). The durations of hospitalization for the neutropenic newborns and 
newborns with normal neutrophils were determined to be 12 and 9 days, respectively. Accordingly, the duration of hospitalization was not significantly correlated with neutropenia $(\mathrm{P}$-value $=0.1)$. Seven neutropenic newborns $(37 \%)$ and 48 non-neutropenic newborns $(16 \%)$ died. There was a significant relationship between mortality rate and neutropenia $(\mathrm{P}$-value $=0.02)$.

\section{Discussion}

In this study, 315 newborns who were admitted to the neonatal intensive care units were evaluated. It should be noted that 19 newborns $(6 \%)$ had neutropenia, which was similar to the results of studies conducted by Balli in the United States $(6 \%)$, Ant in Freiburg et al. in the United States (8\%) [7-9]. In the present study, the neutropenia rates in low-birth-weight newborns and very-low-birth-weight newborns were $7.2 \%$ and $9 \%$, respectively. These statistics were approximately the same as those obtained by Chirico (5.5\% and 7.5\%, respectively) [10]. The mean birth weight of neutropenic newborns ( \pm 448.75) was $1488 \mathrm{~g}$, and the mean birth weight of nonneutropenic newborns (732.32) was $1929 \mathrm{~g}$. Thus, there was a strong and significant correlation between newborn birth weight and neutropenia ( $\mathrm{P}$-value $=0.01)$. A German study found that the level of neutropenia in low-birth-weight newborns was greater than that in normal weight newborns [7].

Regarding the gender of neutropenic newborns, it was found that 14 newborns $(73.4 \%)$ were male and $5(26.3 \%)$ were female. Thus, there was no significant relationship between the gender of newborns and neutropenia ( $p$-value=0.4). VetterLaracy et al. conducted a study on this issue and found that the percentages of male and female newborns in regard to neutropenia were $55 \%$ and $45 \%$, respectively [11]. In general, the percentage of male newborns was higher than that of female newborns.

In this study, $52.6 \%$ of the neutropenic newborns had mothers who had gestational hypertension, and only $15.2 \%$ of the nonneutropenic newborns had mothers with gestational hypertension. There was a strong significant correlation between maternal blood pressure and neonatal neutropenia ( $p$ value=0.000). Queing, Mazinho, Sandra and Christensen each conducted studies in this regard and found that $49 \%, 50 \%$, $32.9 \%$ and $68 \%$ of neutropenic newborns had mothers with gestational hypertension, respectively. The latter findings were consistent with the results of this study $[8,12,13]$. However, Siwa conducted a study in India and found that there was no significant difference regarding maternal blood pressure in neutropenic newborns [14].

The relationship between the five-minute Apgar score and the likelihood of the occurrence of neutropenia was investigated in the two groups (including those newborns with an Apgar score below 5 and those with an Apgar score equal to or greater than 5). In the present study, $10 \%$ of the newborns with an Apgar score below 5 were categorized as neutropenic newborns, which occurred twice as often than in newborns with an Apgar score higher than $5(5.5 \%)$. However, there was no significant relationship between neutropenia in newborns and an Apgar score below and above $5(\mathrm{P}=0.1)$. In general, the five-minute Apgar score and an Apgar score below 5 were associated with neutropenia and neutrophilia, and the likelihood of neutrophilia formation was greater than the latter situation; this was in line with the results of this study [15]. Therefore, it is necessary to re-examine the relationship between asphyxia and neutropenia more precisely and with a larger sample size. The relationship between the above mentioned pairing and an increased or decreased number of neutrophils should be defined more precisely.

In this study, the impact of duration of hospitalization on neutropenic newborns and non-neutropenic newborns was studied. The durations of hospitalization for the neutropenic newborns and non-neutropenic newborns were determined as 12 and 9 days, respectively. There was no significant difference between the duration of hospitalization in newborns in the two groups $(p=0.1)$. However, a three-day difference is very important during hospitalization in terms of clinical and costrelated issues.

The rate of infection in neutropenic newborns was also studied. In the present study, $26.3 \%$ of neutropenic newborns and $12.5 \%$ of non-neutropenic newborns had an early neonatal infection. There was no significant relationship between the groups $(\mathrm{p}=0.86)$. Although Sandra likely found that there was a relationship between neutropenia and infection, it seemed that most neutropenic newborns had necrotizing enterocolitis (NEC) [16]. In another study, 5.5\% of newborns with infections had neutropenia, and there was a significant correlation $(\mathrm{P}=0.001)$ between late infections with Gramnegative bacteria (compared to infection with Gram-positive bacteria) and neutropenia [17]. However, in Paolo's study, there was no significant relationship between neutropenia and infections in very-low-birth-weight newborns [18]. The possible reason for the difference in the present study and several other studies is that those studies linked neutropenia to NEC. Those studies also focused on the clinical symptoms that are used to diagnose neonatal infections. The age of the newborns being evaluated was less than 7 days, but other studies focused on cases of neutropenia associated with late neonatal infections. Since a number of infections can cause neutropenia or neutrophilia and subsequently neutropenia puts newborns at a higher risk of infection $[1,6,19]$, it seems necessary to conduct a more accurate examination with a larger sample size, perform a daily cell count test and use a more precise blood culture method to determine the association between infection and neutropenia.

In this study, the association between mortality rate and neutropenia was investigated; there were 7 cases $(36.8 \%)$ in neutropenic newborns and 48 cases $(16.2 \%)$ in nonneutropenic newborns. Thus, there was a significant relationship between the mortality rates in neutropenic and non-neutropenic newborns $(\mathrm{P}=0.02)$. 


\section{Conclusion}

The results of this study showed that as the gestational age and birth weight of the newborn decrease, the likelihood of neutropenia occurrence increases. There is a strong correlation between maternal hypertension and neutropenia. The mortality rate in neutropenic newborns is also higher than the mortality rate in non-neutropenic newborns. However, there is no significant correlation among neonatal infection, Apgar scores equal to or less than 5 and the gender of newborns with neutropenia. The duration of hospitalization in neutropenic newborns was three days longer than that in non-neutropenic newborns.

\section{Acknowledgements and Appreciation}

This article has been extracted from a dissertation conducted by a student in General Medicine. The authors of the article sincerely thank the Research Deputy of Ahvaz Jundishapur University of Medical Sciences for supporting this study (Registration Code: U-91101).

\section{References}

1. Martin RJ, Fanaroff AA, Walsh MC. Fanaroff and Martins neonatal-perinatal medicine e-book: diseases of the fetus and infant. Elsevier Health Sciences 2010.

2. Maheshwari A, Christensen RD. Neutropenia in the neonatal intensive care unit. NeoReviews 2004; 5: e431e43.

3. Maheshwari A. Neutropenia in the newborn. Curr Opin Hematol 2014; 21: 43-49.

4. Han TH, Chey MJ, Han KS. Granulocyte antibodies in Korean neonates with neutropenia. J Korean Med Sci 2006; 21: 627-632.

5. Procianoy RS, Silveira RC, Mussi-Pinhata MM, Rugolo LMSS, Leone CR, de Andrade Lopes JM. Sepsis and neutropenia in very low birth weight infants delivered of mothers with preeclampsia. J Pediatr 2010; 157: 434-438..

6. Christensen RD. Hematologic problems of the neonates. Saunders 2000.

7. Funke A, Berner R, Traichel B, Schmeisser D, Leititis JU, Niemeyer CM. Frequency, natural course, and outcome of neonatal neutropenia. Pediatrics 2000; 106: 45-51.

8. Christensen RD, Henry E, Wiedmeier SE, Stoddard RA, Lambert DK. Low blood neutrophil concentrations among extremely low birth weight neonates: data from a multihospital health-care system. J Perinatol 2006; 26: 682-687.
9. Maheshwari A, Christensen RD, Calhoun DA. Immunemediated neutropenia in the neonate. Acta Paediatr 2002; 91: 98-103.

10. Chirico G, Motta M, Villani P, Cavazza A, Cardone ML. Late-onset neutropenia in very low birthweight infants. Acta Paediatr 2002; 91: 104-108.

11. Vetter-Laracy S, Balliu PR, Salinas JA, Duran MA. Lateonset neutropenia: defining limits of neutrophil count in very low birth weight infants. J Perinatol 2014; 34: 22-26.

Koenig JM, Christensen RD. Incidence, neutrophil kinetics, and natural history of neonatal neutropenia associated with maternal hypertension. N Engl J Med 1989; 321: 557-562.

12. Mouzinho A, Rosenfeld CR, Sanchez PJ, Risser R. Effect of maternal hypertension on neonatal neutropenia and risk of nosocomial infection. Pediatrics 1992; 90: 430-435.

13. Sivakumar S, Bhat BV, Badhe BA. Effect of pregnancy induced hypertension on mothers and their babies. Indian J Pediatr 2007; 74: 623-625.

14. Fanaroff AAM, Fanaroff RJAA, Martin RJ. Neonatalperinatal medicine: diseases of the fetus and infant. Mosby 2002.

15. Juul SE, Haynes JW, McPherson RJ. Evaluation of neutropenia and neutrophilia in hospitalized preterm infants. J Perinatol 2004; 24: 150-157.

16. Sarkar S, Bhagat I, Hieber S, Donn SM. Can neutrophil responses in very low birth weight infants predict the organisms responsible for late-onset bacterial or fungal sepsis? J Perinatol 2006; 26: 501-505.

17. Manzoni P, Farina D, Monetti C, Priolo C, Leonessa M, Giovannozzi C. Early-onset neutropenia is a risk factor for Candida colonization in very low-birth-weight neonates. Diagn Microbiol Infect Dis 2007; 57: 77-83.

18. Stoll BJ. Infections of the neonatal infant. Textbook Pediatrics 2007; 794-811.

\section{*Correspondence to}

Arash Malakian

Department of Paediatrics

Imam Khomeini Hospital

Ahvaz Jundishapur University of Medical Sciences

Iran 